Original Paper http://ajol.info/index.php/ijbcs http://indexmedicus.afro.who.int

\title{
Caractéristiques morphologique et histologique des gonades de Pseudotolithus elongatus (Bowdich, 1825) de la lagune Ebrié en Côte d'Ivoire
}

\author{
Tiéhoua $\mathrm{KONE}^{1 *}$, Yao Nicolas $\mathrm{AMON}^{1}$, Irène Kessia Fokouo KOUAKOU ${ }^{1}$ et \\ Konan N'DA ${ }^{2}$ \\ ${ }^{1}$ Université Péléforo Gon Coulibaly de Korhogo, UFR Sciences Biologiques, Département de Biologie \\ Animale, BP 1328 Korhogo, Côte d'Ivoire. \\ ${ }^{2}$ Université Nangui Abrogoua, UFR Sciences de la Nature, Laboratoire de Biologie et Cytologie Animales, 02 \\ BP 801 Abidjan 01, Côte d'Ivoire. \\ *Auteur correspondant ; E-mail: ktiehoua@yahoo.fr ; Tel (+225) 0749583661
}

\author{
Received: 07-03-2021 \\ Accepted: 25-05-2021 \\ Published: 30-06-2021
}

\section{RESUME}

Pseudotolithus elongatus est un poisson régulièrement rencontré dans les débarquements de la pêche artisanale et commerciale pratiquée en lagune Ebrié. Cependant, en Côte d'Ivoire aucune étude n'a encore été menée sur cette espèce bien qu'elle ait une valeur commerciale élevée et qu'elle soit beaucoup prisée par la population. Du fait de l'intérêt commercial que présente $P$. elongatus, l'étude de sa reproduction est nécessaire si l'on souhaite assurer la pérennité de l'espèce et satisfaire le consommateur. L'étude de la morphologie et de l'histologie des gonades a été basée sur la taille, la forme, la coloration la présence ou l'absence d'ovocytes mûrs, le diamètre des ovocytes et l'observation histologique. Les résultats obtenus ont révélé six stades de maturation des gonades avec une gamétogenèse continue observée chez cette espèce. L'ensemble de ces informations contribueront à pérenniser l'espèce par une gestion rationnelle du stock.

(C) 2021 International Formulae Group. All rights reserved.

Mots clés : Pseudotolithus elongatus, morphologie, histologie, gonades

\section{Morphological and histological characteristics of the gonads of pseudotolithus elongatus (Bowdich, 1825), from Ebrié lagoon, Côte d'Ivoire}

\section{ABSTRACT}

Pseudotolithus elongatus is a fish regularly met in the landings of the artisanal and commercial fishing on Ebrie lagoon. However, in Ivory Coast no study has been conducted on this species although it has a high commercial value and it is very appreciated by the people. Because of the commercial interest of P. elongatus studying its reproduction is necessary if one wishes to improve operations in order to ensure the sustainability of the species and satisfy the consumer. The studies of gonads morphology and histology of Pseudotolithus elongatus was based on the size, shape, staining of the gonads, the presence or absence of ripe oocytes, the 
diameter of the oocytes and the histological observation. The results obtained indicate six stages of gonad maturation with continuous gametogenesis observed in this species. All of this information will contribute to perpetuate this species through rational management of the stock.

(C) 2021 International Formulae Group. All rights reserved.

Keywords: Pseudotolithus elongatus, morphology, histology, gonads.

\section{INTRODUCTION}

Les lagunes sont des foyers d'occupation humaine en raison de leurs fortes ressources halieutiques. Elles sont l'objet de pressions liées aux méthodes modernes d'exploitation des ressources vivantes (Dufour et al., 1994). La lagune Ebrié est l'une des lagunes les plus exploitées du système lagunaire ivoirien. La proximité géographique de celle-ci avec des secteurs fortement peuplés tel que la zone périurbaine d'Abidjan induit une exploitation considérable de ses ressources. Face à tous ces fléaux, il s'avère donc nécessaire d'entreprendre un plan de gestion durable des ressources afin de préserver l'équilibre écologique des écosystèmes aquatiques (Boussou, 2013).

En lagune Ebrié, la pêche artisanale s'organise autour de l'exploitation des crustacés et mollusques d'une part, et de celle des poissons dont les captures sont dominées essentiellement par certaines familles telles que les Clupeidae, les Claridae, les Carangidae, les Cichlidae, les Cugilidae, les Sciaenidae, les Claroteidae (Durand et al., 1994 ; Paugy et al., 2004).

La famille des Sciaenidae constitue la communauté de poissons la plus importante du golfe de Guinée (Teitelbaum, 1999). Cette famille compte environ 270 espèces réparties en 70 genres environ (Chao et al., 2001; Chao, 2003) dont les principaux représentants sont le genre Pseudotolithus. Sur les côtes Ouestafricaines, huit genres repartis en seize espèces sont reconnues. Parmi ces espèces, seulement trois appartenant au genre Pseudotolithus sont jusqu'à lors rencontrées en eaux saumâtres (Albaret, 1994). Ce sont Pseudotolithus elongatus, Pseudotolithus senegalensis et Pseudotolithus typus.

Communément appelé "Sosso", Pseudotolithus elongatus est très rentable dans le secteur de la pêche grâce à sa valeur commerciale élevée et à ses qualités organoleptiques. Malgré cet intérêt accru aussi bien de la part des consommateurs que des pêcheurs, les études sur la reproduction sont inexistantes dans les eaux ivoiriennes.

Dans cette étude préliminaire sur la reproduction de Pseudotolithus elongatus, il sera présenté ici une échelle de développement macroscopique des ovaires suivie d'un volet microscopique où les stades évolutifs des cellules germinales seront décrits au cours de l'ovogenèse.

\section{MATERIEL ET METHODES Milieu d'étude}

Cette étude a été menée sur des spécimens de Pseudotolithus elongatus pêchés dans la lagune Ebrié. C'est une lagune située dans le Sud de la Côte d'Ivoire. Elle est limitée à l'Ouest par le canal d'Assagny et à l'Est par le canal d'Assinie. C'est un système lagunaire de forme allongée avec une superficie de 566 $\mathrm{km}^{2}$ (Varlet, 1978). Située entre les longitudes $3^{\circ} 47^{\prime}$ et $5^{\circ} 29^{\prime}$ Ouest et les latitudes $5^{\circ} 02^{\prime}$ et $5^{\circ} 42^{\prime}$ Nord, la lagune a environ $130 \mathrm{~km}$ de longueur et une largeur maximum de $7 \mathrm{~km}$ (Albaret, 1994 ; Durand et Guiral, 1994 ; Lae, 1997). Le volume total de la lagune est d'environ 2,7109 $\mathrm{m}^{3}$. La profondeur moyenne de l'eau est de 4,8 m (Tastet et Guiral, 1994). Séparée de l'Océan Atlantique par un cordon littoral sableux, la lagune Ebrié est traversée en sa partie centrale par le canal de Vridi. D'ailleurs, le canal de Vridi constitue la seule voie de communication de la lagune avec la mer. La lagune Ebrié reçoit aussi de l'eau douce venant de la Comoé, de l'Agnéby et de la Me (Lae, 1992 ; Durand et al., 1994).

La zone d'étude correspond au secteur III de la lagune. Il s'agit de la zone estuarienne qui englobe toute la zone urbaine d'Abidjan et le canal de Vridi. Cette zone est caractérisée par l'influence directe des eaux marines. Selon Domain et al. (2000a) les Sciaenidae sont reparti en deux groupes. Il s'agit des Sciaenidae côtiers et des Sciaenidae estuariens. Pseudotolithus elongatus appartenant au 
groupe des Sciaenidae estuariens, le secteur III de la lagune a été choisi comme zone d'étude (Figure 1).

\section{Matériel biologique et méthodes Matériel biologique}

Les poissons ayant servi à cette étude ont été collectés mensuellement de juillet 2013 à juin 2015 au niveau de la zone III de la lagune Ebrié. Ainsi, un total de 550 spécimens capturés à l'aide de filets maillants (mailles: comprise entre 20 et $40 \mathrm{~mm}$ ) ont été identifié selon les clés de détermination de Paugy et al. (1992).

\section{Méthodes}

Pour chaque spécimen, la longueur standard, la longueur totale ainsi que le poids vif et le poids éviscéré ont été respectivement relevés au millimètre et au gramme près. $\mathrm{La}$ détermination du sexe a été faite par l'observation directe des gonades après dissection du poisson. Les gonades et le foie ont été isolés et pesés au gramme près.

Le stade macroscopique de maturité sexuelle a été déterminé selon une échelle inspirée de Fontana (1969). Cette échelle tient compte de l'aspect macroscopique des glandes à savoir la forme, la couleur, la vascularisation, la réaction à la pression et de la taille des ovocytes.

La technique utilisée pour l'étude microscopique ou histologique des gonades de Pseudotolithus elongatus est celle proposée par Martoja et Martoja (1967).

Après la dissection et prélèvement des organes, trois petits fragments ont été prélevés, l'un dans la partie médiane et les deux autres aux extrémités. Ceci a permis de déterminer le stade de maturité sexuel avec plus de précision;

Ces organes ont été fixés immédiatement dans une solution de formol a $10 \%$ pendant 2 à 3 jours dans des piluliers et mis ensuite dans de l'alcool à $70^{\circ}$;

- Les pièces ont été ensuite déshydratées dans des bains d'alcool de degrés croissants;

- Apres la déshydratation, les organes ont été passés dans 3 bains successifs de toluène;

- Des séries de coupes de $7 \mu \mathrm{m}$ d'épaisseur sont effectuées au microtome. Les coupes ont ensuite été soumises au déparaffinage et à la déshydratation. Les coupes ont enfin été plongées dans du toluène;

Les observations et les mesures des diamètres du noyau et du cytoplasme ont été faites à l'aide d'un microscope muni d'un micromètre.

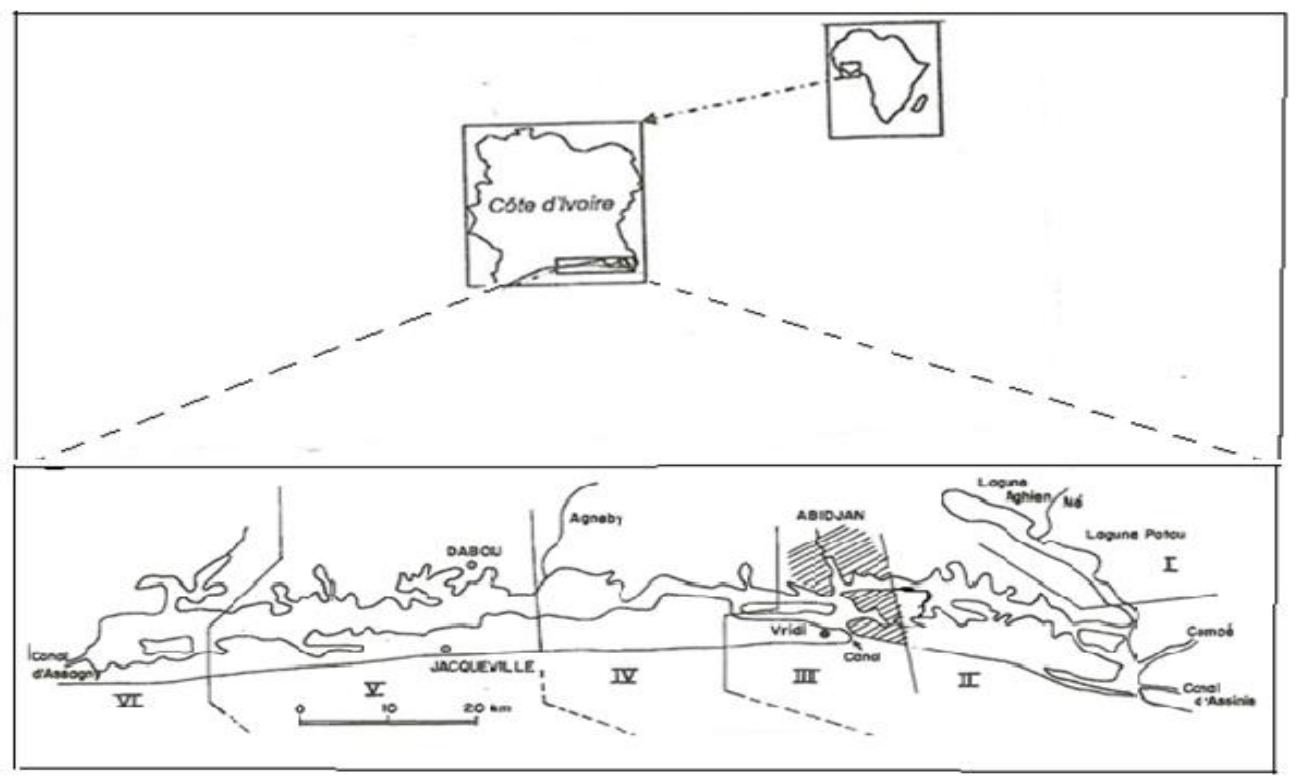

Figure 1 : Subdivision de la lagune Ebrié en secteurs selon Durand et Skubich (1982) et Durand et Guiral (1994).

: Zone d'étude 
RESULTATS

\section{Caractéristiques gonades \\ morphologiques des}

Pseudotolithus elongatus présente une gonade à deux lobes. La gonade femelle est cylindrique et de couleur orange tandis que chez le mâle, elle est plate et présente une couleur blanchâtre.

\section{Evolution des stades macroscopiques des ovaires}

\section{Description des gonades}

La description des différents aspects macroscopiques de Pseudotolithus elongatus est la suivante :

Stade I ou Immature (Figure 1a) : La gonade est ferme, transparente ou rose-clair et de petite taille. Elle est filamenteuse avec une membrane très fine. Les ovocytes ne sont pas visibles.

Stade II ou Début de développement (Figure 1b) : L'ovaire devient opaque et ferme. L'ovaire passe du rose pâle à l'orange clair. Les ovocytes sont invisibles à l'œil nu.

Stade III ou Vitellogénèse primaire (Figure 1c): Les ovocytes sont toujours invisibles mais l'ovaire prend une couleur orange clair.

Stade IV ou Vitellogénèse secondaire (Figure 1d) : L'ovaire est très gros avec une membrane ovarienne est très fine. Les ovocytes sont visibles à travers la membrane ovarienne. L'ovaire prend une couleur orange foncé.

Stade V ou Ponte (Figure 1e) : L'ovaire est très gros et les ovocytes sont bien visibles. A la moindre pression exercée sur l'abdomen les ovocytes sont expulsés. L'ovaire est de couleur orange foncé.

Stade VI ou Post-ponte (Figure 1f): L'ovaire est flasque, très vascularisé et très mou. Il a l'aspect d'un sac vide et possède une coloration rouge due à une très forte vascularisation.

\section{Caractéristiques microscopiques des gonades \\ Ovogenèse}

Les étapes de l'évolution microscopique des ovocytes sont classées en six stades :
Stade I ou ovocytes primaire (Figure 2a): Les Ovocytes sont de petite taille, de forme polyédrique avec un noyau délimité par l'enveloppe nucléaire. Les nucléoles sont disposés contre la membrane nucléaire. Le diamètre des ovocytes est compris entre $30 \mu \mathrm{m}$ et $70 \mu \mathrm{m}$ et celui du noyau compris entre $20 \mu \mathrm{m}$ et $30 \mu \mathrm{m}$.

Stade II ou ovocytes prévitellogénique (Figure $2 \mathrm{~b}$ ) : Les ovocytes sont de taille plus grande avec un gros noyau. Le cytoplasme est plus volumineux. Les nucléoles sont disposés à la périphérie du noyau. Le diamètre des ovocytes est compris entre 50 et $170 \mu \mathrm{m}$ et celui du noyau compris entre 20 et $70 \mu \mathrm{m}$.

Stade III ou ovocytes en vitellogénèse primaire (Figure 2c): C'est le début de la vitellogénèse avec apparition et développement d'une couronne de granules vitellins de la périphérie de la cellule vers le noyau. Le vitellus occupe tout l'espace protoplasmique à l'exception de deux zones cytoplasmiques une à la périphérie de la cellule et l'autre autour du noyau. Le diamètre des ovocytes est compris entre 80 et $340 \mu \mathrm{m}$ et le diamètre du noyau entre 30 et $190 \mu \mathrm{m}$.

Stade IV ou ovocytes en vitellogénèse secondaire (Figure 2d) : Les ovocytes sont de grande taille et de forme arrondie. La quantité de vitellus augmente dans le cytoplasme. Le noyau est en position centrale et la taille des ovocytes varie de 190 à $780 \mu \mathrm{m}$. Le diamètre du noyau est compris entre 50 et $240 \mu \mathrm{m}$.

Stade $\mathrm{V}$ ou ovocytes en vitellogénèse tertiaire ou maturation (Figure 2e) : Le volume cellulaire est envahi par les inclusions vitellines. Des vacuoles de différentes tailles apparaissent entre les grains de vitellus. Le noyau est excentré avec apparition d'une grosse gouttelette lipidique occupant le centre de l'ovocyte. Les ovocytes ont une taille comprise entre 192 et $810 \mu \mathrm{m}$; et le diamètre du noyau compris entre 60 et $130 \mu \mathrm{m}$.

Stade VI ou ovocytes hyalins (Figure 2f) : L'ovaire après la ponte apparait nettement désorganisé. Il y'a une coalescence des inclusions vitellines et des vacuoles. Tous les ovocytes en voie de maturation présentent des signes de nécrose. 


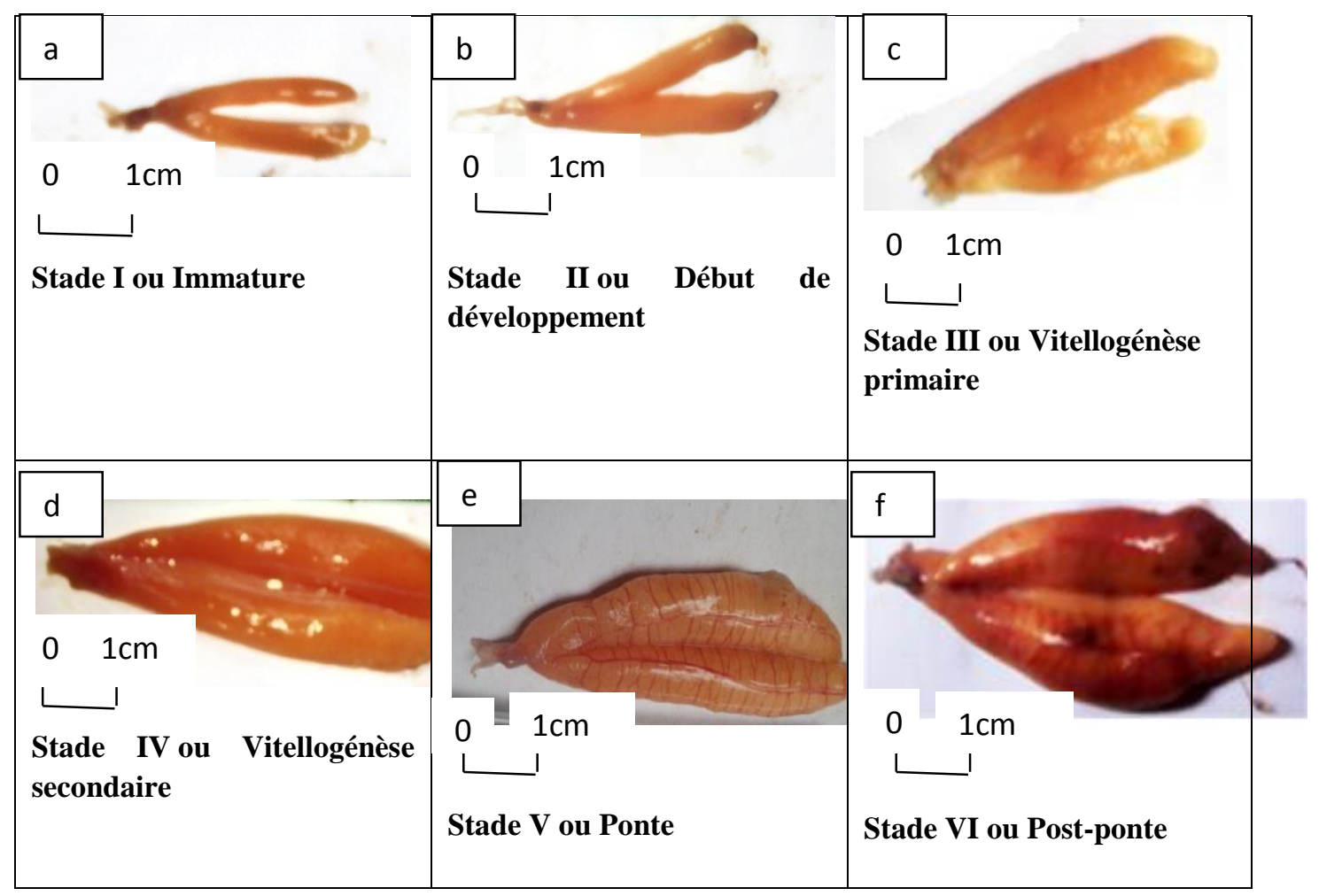

Figure 1: Evolution des stades macroscopiques des ovaires de Pseudotolithus elongatus.

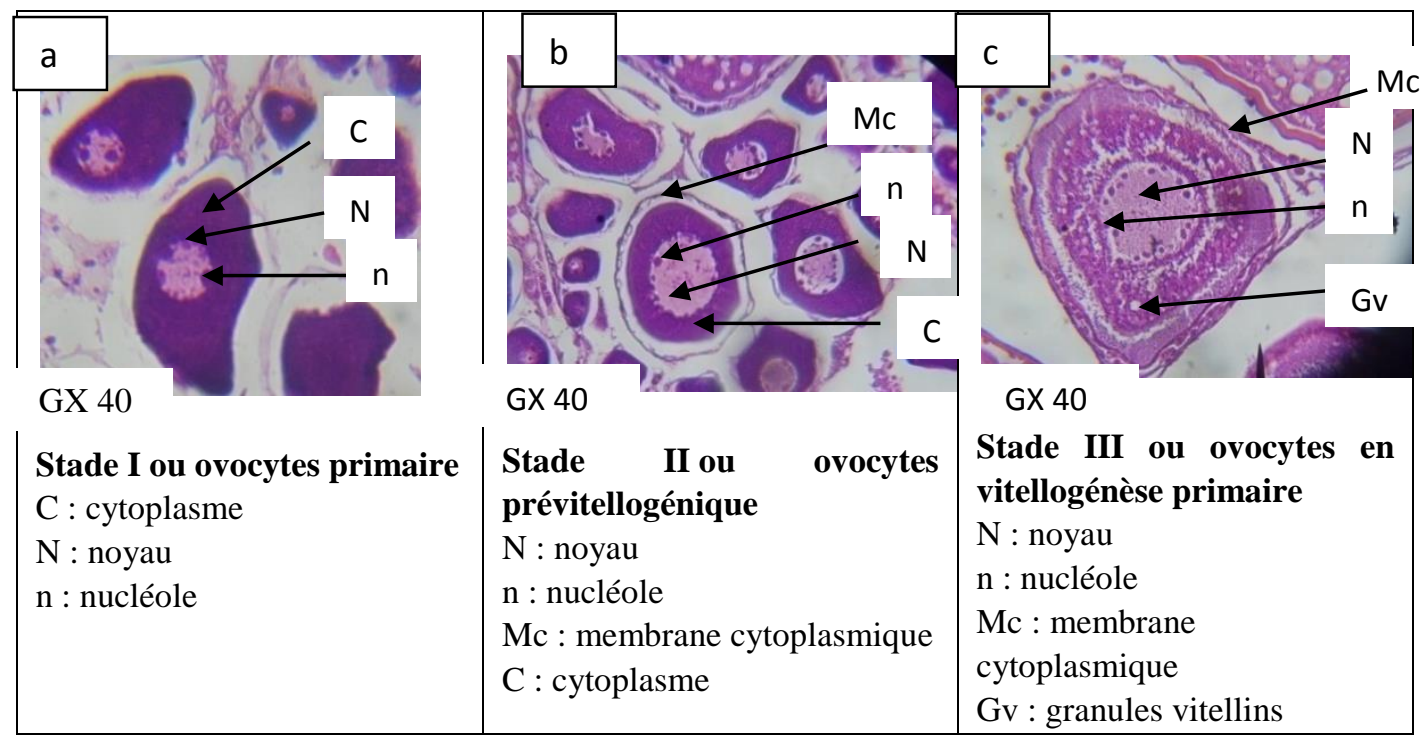




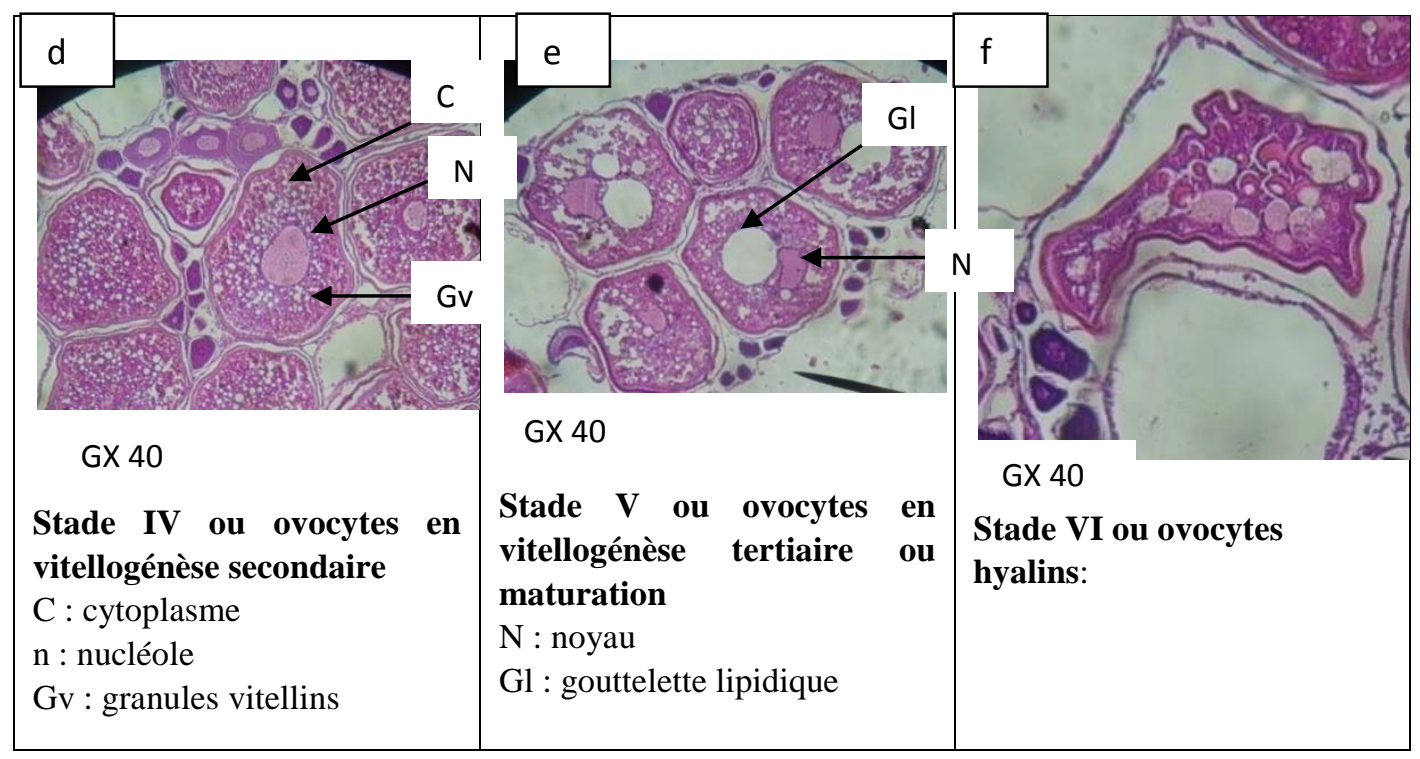

Figure 2: Evolution des stades microscopiques dans l'ovogenèse de Pseudotolithus elongatus.

\section{DISCUSSION}

L'examen macroscopique des ovaires de Pseudotolithus elongatus a permis d'obtenir six stades. Dans les gonades, tous les ovocytes n'atteignent pas la maturité simultanément. Ce qui suggère que la gamétogenèse chez $P$. elongatus est continue. La présence d'ovocytes de différents stades dans le même ovaire témoigne de l'émission des produits génitaux tout le long de l'année. Nos résultats sont en accord avec ceux de Domain et al. (2000b) réalisés sur $P$. elongatus en Guinée. Selon ces auteurs la reproduction de cette espèce a lieu toute l'année. $\mathrm{La}$ reproduction de Pseudotolithus elongatus toute l'année a également été rapportée par Enakem et al. (2004) dans la rivière Cross au Nigeria. Toutefois, ces auteurs ont remarqué que l'activité sexuelle de cette espèce est plus intense de décembre à février.

\section{Conclusion}

Ce travail a été entrepris dans le but d'étudier la reproduction de Pseudotolithus elongatus; notamment les différents stades de maturation macroscopiques et microscopiques des gonades. Six stades de développement (stades I, II, III, IV, V et VI) ont été observés. Le développement des ovocytes se fait de façon asynchrone et ceux-ci n'atteignent pas la maturité simultanément. Des ovocytes de différents stades sont retrouvés dans les gonades pendant la période de reproduction.

\section{CONFLITS D'INTERETS}

Les auteurs déclarent qu'ils n'ont aucun conflit d'intérêts.

\section{CONTRIBUTIONS DES AUTEURS}

TK a fait non seulement les prélèvements, mais aussi, a contribué à l'analyse des échantillons et à la rédaction de la version initiale du manuscrit. YNA a contribué à la réalisation des coupes histologiques. IKFK a aidé à l'identification des différents stades microscopiques des gonades. $\mathrm{KN}$ a supervisé tous les travaux et a révisé le manuscrit.

\section{REMERCIEMENTS}

Les auteurs tiennent à remercier le Directeur du Laboratoire de Biologie et Cytologie Animales de l'Université Nangui Abrogoua pour avoir facilité les analyses.

\section{REFERENCES}

Albaret JJ. 1994. Les poissons, biologie et peuplement. In Environnement et ressources aquatiques en Côte d'Ivoire: 
Les milieux lagunaires, Durand JR, Dufour Ph, Guiral D, Zabi SGF (Eds). ORSTOM: $\quad$ Paris; 239-280. http://www.documentation.ird.fr/hor/fdi: 40693

Boussou KC. 2013. Variations morphométriques, biologie et écologie du Cichlidae Chromidotilapia guntheri (Sauvage, 1882) dans des hydrosystèmes de l'Est et du Sud de la Côte d'Ivoire. Thèse de Doctorat, Université Nangui Abrogoua, p. 161.

Chao NL. 2003. Sciaenidae. In The living marine resources of the western central Atlantic, species identification guide for fishery purposes, Carpenter KE (Eds). Document FAO, 3(2): 1583-1653. http://www.fao.org/3/y4162e/y4162e00. $\mathrm{htm}$

Chao NL, Bearez P, Robertson DR. 2001. A new genus and new species of Sciaenidae from the Gulf of Panama (Perciformes: Sciaenidae). Revue de Biologie Tropicale, 49(1): 81-88.

http://www.academia.edu/5620698/

Domain F, Keita M, Morize E. 2000a. Typologie générale des ressources demersales du plateau continental. In $L a$ pêche côtière en Guinée - Ressources et Exploitation, Domain F, Chavance P, Diallo A (eds). IRD/CNSHB: Paris; 5385. https://agris.fao.org/agris/

Domain F, Chavance P, Bah A. 2000b. Notes sur la reproduction des espèces démersales du peuplement côtier guinéen. In La Pêche Côtière en Guinée Ressources et Exploitation, Domain F, Chavance P, Diallo A (eds). IRD/CNSHB, p.393. http://www.documentation.ird.fr/hor/fdi: 010025015

Dufour P, Lemoalle J, Albaret JJ. 1994. Le système Ebrié dans les typologies lagunaires. In Environnement et Ressources Aquatiques de Côte d'Ivoire Les Milieux Lagunaires, Durand JR, Dufour P, Guiral D, Zabi SGF (eds). ORSTOM : $\quad$ Paris ; 1724. https://www.documentation.ird.fr/ho r/fdi:40683.
Durand JR, Guiral D. 1994. Hydroclimat et hydrochimie. In Environnement et Ressources Aquatiques de Côte d'Ivoire Les Milieux Lagunaires, Durand JR, Dufour P, Guiral D, Zabi SG (eds). ORSTOM : $\quad$ Paris ; 5990. http://horizon.documentation.ird.fr/e $\mathrm{xl}-$ doc/pleins_textes/pleins_textes_7/divers 2/40686

Durand JR, Dufour P, Guiral D, Zabi SG. 1994. Environnement et ressources aquatiques de Cote d'Ivoire. Les milieux lagunaires, p.546.

https://horizon.documentation.ird.fr/exld oc/pleins_textes/pleins_textes_7/divers2/ 37706

Durand JR, Skubich .1982. Les lagunes ivoiriennes. Aquaculture, 27: 211- 250. DOI: $\quad$ https://doi.org/10.1016/00448486(82)90059-X

Enakem SB, Achima MJ, Ekere NM. 2004. Studies of some reproductive aspect of Pseudotolithus elongatus in the Cross River estuary, Nigeria. Scientia Marina, 68(2): 265-271. DOI : https://doi.org/10.3989/scimar.2004.68n 2265

Fontana A. 1969. Etude de la maturité sexuelle des sardinelles Sardinella eba et Sardinella aurita de la région de PointeNoire. ORSTOM, 7(2): 101-114. https://agris.fao.org/agrissearch/search.do?recordID=AV2012086 266

Fontana A, Le Guen JC. 1969. Etude de la maturité sexuelle et de la fécondité de Pseudotolithus (fonticulus) elongatus. ORSTOM, 7(3): 919. http://horizon.documentation.ird.fr/e $\mathrm{xl}-$

doc/pleins_textes/cahiers/oceanographie/ 19562

Lae R. 1997. Does overfishing lead to a decrease in catches and yields? An example of two West African Coastal Lagoons. Fisheries Management and Ecology, 4: 149164. http://horizon.documentation.ird.fr/ exl- 
doc/pleins_textes/pleins_textes_6/b_fdi_ 49-50/010014114

Lae R. 1992. Les pêcheries artisanales lagunaires Ouest africaines : échantillonnage et dynamique de la ressource et de l'exploitation. Thèse de Doctorat, Université de Bretagne occidentale, p. 201. http://horizon.documentation.ird.fr/exldoc/pleins_textes/divers14-08/31385.pdf Martoja R, Martoja PM. 1967. Initiation aux Techniques de l'Histologie Animale. Masson et Cie 120: bd St-Germain, Paris-VIe, p. 345. https://bibliotheques.mnhn.fr/medi as/doc/EXPLOITATION/HORIZON/36 $131 /$

Paugy D, Lévêque C, Teugels GG. 1992. Faune des poissons d'eaux douces et saumâtres de l'Afrique de l'Ouest, Tome II. p.902. http://horizon.documentation.ird.fr/exldo c/pleins_textes/pleins_textes_6/Fau_trop /34060/

Tastet JP, Guiral D. 1994. Géologie et sédimentologie. In Durand JR, Dufour P,
Guiral D, Zabi SGF (eds). Environnement et Ressources Aquatiques de Côte d'Ivoire. Les Milieux Lagunaires : Paris ; 35-57.

https://www.documentation.ird.fr/hor/fdi :40685

Teitelbaum A. 1999. Ecologie des communautés de poissons en Guinée. Exemple de la communauté a Sciaenidae dans les îles de Loos. Rapport de Stage, Ecole Supérieure d'Agro-Economie International (ISTOM), IRD-CNSHB : France ; p.60. http://horizon.documentation.ird.fr/exldoc/pleins_textes/divers1710/010043889.pdf

Varlet F. 1978. Le régime de la lagune Ebrié (Côte d'Ivoire). Traits physiques essentiels, ORSTOM, p. 164. https://www.documentation.ird.fr/hor/fdi :09308. 